\title{
Routine analysis of cirrhotic ascites for evidence of infection - not worth the effort?
}

\author{
Varghese Thomas
}

Received: 29 August 2011 / Accepted: 23 September 2011 /Published online: 11 October 2011

(C) Indian Society of Gastroenterology 2011

Spontaneous bacterial peritonitis (SBP) is a serious complication of advanced cirrhosis of the liver. Usually patients with SBP show symptoms such as fever, abdominal pain, worsening of renal function, hypotension or development of encephalopathy. The frequency of SBP among hospitalised patients with advanced cirrhosis varies from $10 \%$ to $30 \%$ [1]. The mortality of SBP was $80 \%$ to $100 \%$ in the 1960 s, but has declined to $30 \%$ to $40 \%$ with early diagnosis and effective therapy with broad-spectrum antibiotics $[2,3]$. A high index of suspicion followed by analysis of ascitic fluid for evidence of infection is helpful in making an early diagnosis of SBP, and is today considered the standard of care in patients with cirrhosis and symptoms listed above. The International Ascites Club recommends mandatory analysis of ascitic fluid in all cases of new onset of ascites, worsening of ascites, and in all other cases whenever there is a suspicion of SBP [1].

The recommendations of the American Association for the Study of Liver Disease (AASLD) differ from those of the International Ascites Club. The AASLD thus recommends testing of ascitic fluid for cell count and differential count, but not necessarily culture, for patients undergoing serial outpatient therapeutic paracentesis, each time the fluid is removed [4]. Given the low prevalence of SBP in asymptomatic patients, the question is whether we need to undertake ascitic fluid cell count routinely in all cases of paracentesis. This issue is of particular importance for gastroenterologists and physicians in India and other developing countries, since such testing entails extra cost.

\footnotetext{
V. Thomas $(\bowtie)$

Department of Gastroenterology, Calicut Medical College, Kozhikode 673 008, India

e-mail: drvarghesethomas@gmail.com
}

Based on the results of absolute white cell count and culture of the ascitic fluid, five variants of peritoneal fluid infection have been recognised [1]. These are: (1) SBP: absolute count of polymorphonuclear leucocytes (PMN) in ascitic fluid $>250 / \mathrm{mm}^{3}$ with a positive culture showing a single type of bacteria; (2) culture-negative neutrocytic ascites (CNNA): negative ascitic fluid culture with PMN count of $>250 / \mathrm{mm}^{3}$; (3) mono-microbial non-neutrocytic bacterascites (MNB): ascitic fluid culture positive for one type of bacteria and PMN count of $<250 / \mathrm{mm}^{3}$; (4) secondary bacterial peritonitis: characterized by polymicrobial growth from ascitic fluid with PMN $>250 / \mathrm{mm}^{3}$ and a surgicallytreatable source of infection; and, (5) polymicrobial bacterascites with PMN count $<250 / \mathrm{mm}^{3}$.

SBP may sometimes be asymptomatic or present with atypical symptoms. In one study, clinical suspicion based on presence of symptoms of SBP in an emergency room setting had a sensitivity of only $76 \%$ and specificity of $34 \%$ for the detection of SBP [5]. This suggested that clinical assessment by itself is inaccurate for the diagnosis or exclusion of SBP, and led the authors to recommend routine ascitic fluid analysis [5].

However, in several studies conducted among outpatients with cirrhosis, the frequency of asymptomatic infection of ascitic fluid has been reported to be as low as $0-3.7 \%$ [6-8]. In this issue of the Journal, Mohan and Venkataraman have published data on asymptomatic infection of ascitic fluid in cirrhotic outpatients from an institution in southern India [9]. They found a low prevalence $(2.5 \%)$ of asymptomatic ascitic fluid infection in their patients; this included asymptomatic SBP in $0.4 \%$, CNNA in $1.4 \%$ and MNB in $0.7 \%$ of their cases. In this study, the mean ascitic fluid protein in the SBP group was comparable with the group with no infection (1.1 [0.1] vs. $1.2[0.2] \mathrm{g} / \mathrm{dL})$. The authors could not find any specific 
risk factors associated with asymptomatic infection of ascitic fluid such as age, gender, etiology and severity of cirrhosis, or ascitic fluid protein levels.

In a recent similar study from Poland, KasztelanSzczerbinska et al. found that $5.4 \%$ of their inpatients with cirrhosis had evidence of asymptomatic SBP, $16.2 \%$ had bacterascites and $2.7 \%$ had CNNA [10]. These rates of ascitic fluid infection were higher than those in the previous studies. This was possibly accounted for by the fact that this Polish study included subjects who were inpatients. Further, these investigators could not identify any specific risk factors for the development of asymptomatic ascitic fluid infection. However, they found that levels of Creactive protein (CRP) in serum were significantly higher in their patients with ascitic fluid infection. Thus, the authors suggested that serum CRP could be used as a marker for presence of ascitic fluid infection. This interesting observation needs further evaluation. Notably, none of the patients in the Polish study died during a 30-day follow up period. The authors therefore believed that asymptomatic infection of ascitic fluid has a milder disease course and has fewer serious consequences when compared with symptomatic SBP.

Another factor that needs consideration is the profile of organisms causing SBP. In the study by Mohan and Venkataraman, two-thirds of bacterial isolates were gramnegative bacilli and one-third were gram-positive cocci. In contrast, in the Polish study, the predominant organisms isolated were gram-positive cocci. This observation was interesting in that gram-negative bacilli have been the most predominant organisms isolated in cases of SBP world over. Some recent reports have described a change in the bacteriological profile of SBP to gram-positive organisms, especially in those who were on long-term prophylaxis against SBP with norfloxacin [11].

Treatment of asymptomatic infection of ascitic fluid also needs consideration. The International Ascites Club recommends that all cases of SBP as also those with CNNA, whether symptomatic or asymptomatic, should be treated with broad spectrum antibiotics [1]. In patients with CNNA, other causes of PMN leukocytosis of ascitic fluid should be excluded (such as previous antibiotic therapy, hepatocellular carcinoma, peritoneal carcinomatosis, tuberculosis, pancreatitis and bleeding). This recommendation is based on the observation that about one-third of untreated cases with CNNA developed a positive ascitic fluid culture during follow up [12]. The symptoms and mortality of patients with CNNA are similar to those of SBP. Further, $33 \%$ to $57 \%$ of patients with CNNA also showed a positive blood culture, providing evidence in support of presence of a systemic bacterial infection [12]. In contrast with CNNA, no standard treatment recommendations exist for MNB. It is believed that nearly $80 \%$ of cases with MNB do not progress to SBP [12]. In such cases, a repeat analysis of ascitic fluid $48 \mathrm{~h}$ later can safely rule out SBP. If there is no increase in cell count, the patient can be followed up without antibiotics. However further studies on natural history of MNB are necessary before any conclusive recommendations can be made.

In most laboratories, ascitic fluid cell count is done using manual techniques. This is time-consuming and liable to a high error rate. Hence, automated cell counts are ideal. Most of the laboratories are not willing to use automated cell counters for ascitic fluid count since the manufacturers of these equipment do not recommend their use for counts on any fluid other than blood. It will be of a great help to clinicians if all laboratories provided automated cell counts in cases of suspected infection of body fluids. The results will be obtained faster, will be more accurate and cheaper. Another option is to use leukocyte esterase reagent (LER) strips for the bedside diagnosis of SBP [13]. LER technique has been shown to be cheaper, faster (it takes less than a minute) and well reproducible [14]. If LER test is positive, the fluid should be immediately inoculated into culture medium and another sample sent for cell count. The first dose of antibiotic can also be given immediately after a positive LER test. However, the clinicians have not shown much enthusiasm in adopting this technique, which could also do with further standardisation.

As indicated above, all previous studies which looked for evidence of asymptomatic infection of ascitic fluid in outpatients with decompensated cirrhosis of the liver had shown only a low prevalence rate. If we exclude MNB since it does not carry a prognosis worse than cirrhotic ascites per se, the prevalence of asymptomatic infection (SBP and CNNA together) will be expected to be even lower. Hence routine testing of ascitic fluid for evidence of infection is not indicated in outpatients with no symptoms or signs of infection. In keeping with this, some published reports state that routine culture of ascitic fluid in asymptomatic patients serves only to add to the treatment costs of patients with cirrhosis and should be avoided [15, 16]. Testing of ascitic fluid for infection is thus likely to be most useful in cases with new onset of ascites, worsening of ascites and in cases where there is a clinical pointer that raises suspicion of SBP. In such patients with a higher likelihood of ascitic fluid infection, we can further improve the detection rates of SBP and its congeners by applying the newer and more rapid tools. In addition, we need to undertake studies to better understand the natural history of SBP, CNNA and MNB, and the need for treatment in patients with asymptomatic ascitic fluid infection. 


\section{References}

1. Rimola A, García-Tsao G, Navasa M, et al. Diagnosis, treatment and prophylaxis of spontaneous bacterial peritonitis: a consensus document. International Ascites Club. J Hepatol. 2000;32:142-53.

2. Hoefs JC, Runyon BA. Spontaneous bacterial peritonitis. Dis Mon. 1985;31:1-48.

3. Khan J, Pikkarainen P, Karvonen AL, et al. Ascites: aetiology, mortality and the prevalence of spontaneous bacterial peritonitis. Scand J Gastroenterol. 2009;44:970-4.

4. Runyon BA. Management of adult patients with ascites caused by cirrhosis. Hepatology. 2004;39:841-56.

5. Chinnok B, Afarian H, Minnigan H, Butler J, Henday GW. Physician clinical impression does not rule out spontaneous bacterial peritonitis in patients undergoing emergency department paracentesis. Ann Emerg Med. 2008;52:268-73.

6. Evans LT, Kim WR, Poterucha JJ, Kamath PS. Spontaneous bacterial peritonitis in asymptomatic outpatients with cirrhotic ascites. Hepatology. 2003;37:897-901.

7. Jeffries MA, Stern MA, Gunaratnam NT, Fontana RJ. Unsuspected infection is infrequent in asymptomatic outpatients with refractory ascites undergoing therapeutic paracentesis. Am J Gastroenterol. 1999;94:2972-6.

8. Romney R, Mathurin P, Ganne-Carrie N, et al. Usefulness of routine analysis of ascitic fluid at the time of therapeutic paracentesis in asymptomatic outpatients. Results of a multicenter, prospective study. Gastroenterol Clin Biol. 2005;29:275-9.
9. Mohan P, Venkataraman J. Prevalence and risk factors for unsuspected spontaneous ascitic fluid infection in cirrhotics undergoing therapeutic paracentesis in an outpatient clinic. Indian J Gastroenterol. 2011;30: doi:10.1007/s12664-011-0131-7.

10. Kasztelan-Szczerbinska B, Słomka M, Celinski K, Serwacki M, Szczerbinski M, Cichoz-Lach H. Prevalence of spontaneous bacterial peritonitis in asymptomatic in patients with decompensated liver cirrhosis - a pilot study. Adv Med Sci. 2011;56:13-7.

11. Park MK, Lee JH, Byun YH, et al. Changes in the profiles of causative agents and antibiotic resistance rate for spontaneous bacterial peritonitis: an analysis of cultured microorganism in recent 12 years. Korean J Hepatol. 2007;13:370-7.

12. Lata J, Stiburek O, Kopacova M. Spontaneous bacterial peritonitis: a severe complication of liver cirrhosis. World J Gastroenterol. 2009; 15:5505-10.

13. Xia HH. Spontaneous bacterial peritonitis. World J Gastroenterol. 2009;15:1042-9.

14. Balagopal SK, Sainu A, Thomas V. Evaluation of leucocyte esterase reagent strip test for the rapid bedside diagnosis of spontaneous bacterial peritonitis. Indian J Gastroenterol. 2010; 29:74-7.

15. Stern MA, Chalasani N, Strauss RM. Is it cost effective or necessary to routinely analyze ascitic fluid in an asymptomatic outpatient population of cirrhotics (abstract)? Hepatology. 1994; 19:1271A.

16. Kolle L, Ortiz J, Ricart E, et al. Ascitic fluid culture is not necessary in asymptomatic cirrhotic outpatients undergoing repeated therapeutic paracentesis (abstract). Hepatology. 1996;24:445A. 